\title{
Why are Individuals with Alexithymia Symptoms More Likely to Have Mobile Phone Addiction? The Multiple Mediating Roles of Social Interaction Anxiousness and Boredom Proneness
}

\author{
Weilong Xiao ${ }^{1,2, *}$ \\ Hui Zhou ${ }^{3} *$ \\ Xinwei $\mathrm{Li}^{\prime}$ \\ Xue $\operatorname{Lin}\left(D^{4}\right.$ \\ 'School of Teacher Education, Zhejiang \\ Normal University, Jinhua, People's \\ Republic of China; ${ }^{2}$ School of Education, \\ Fujian Polytechnic Normal University, \\ Fuzhou, People's Republic of China; \\ ${ }_{3}^{3}$ Jinhua Advanced Research Institute, \\ Jinhua, People's Republic of China; \\ ${ }^{4}$ Psychological Counseling Center of \\ Student Affairs Office, Zhangzhou City \\ College, Zhangzhou, People's Republic of \\ China \\ *These authors contributed equally to \\ this work
}

\begin{abstract}
Purpose: Previous studies have investigated the relationship between alexithymia and problematic mobile phone use (PMPU). However, yet gaps in identifying the internal mechanisms of this relationship remain. Hence, based on the Interaction of Person-AffectCognition-Execution model, the current research examined the mediating roles of college students' social interaction anxiousness (SIA) and boredom proneness (BPS) in the relationship between alexithymia and PMPU.
\end{abstract}

Methods: A total of 1300 college students $\left(M_{\text {age }}=20.36\right.$ years, $\left.S D=0.97\right)$ were recruited from two universities in the southeast of China to complete a series of questionnaires, including the Toronto alexithymia scale, SIA scale, BPS scale, and mobile phone addiction index questionnaire. In order to conduct conservative predictions, the demographic variables (ie, gender) were controlled as covariates.

Results: The results of multiple mediation analysis showed that (1) alexithymia was positively linked with PMPU; (2) both SIA and BPS mediated the link between alexithymia and PMPU; and (3) a serial indirect pathway emerged (ie, alexithymia $\rightarrow$ SIA $\rightarrow$ BPS $\rightarrow$ PMPU).

Conclusion: These findings indicated that alexithymia could influence PMPU in a simple indirect way (parallel mediation) and in a complex indirect way (serial mediation). Besides, these findings provide some insights into the prevention and intervention of PMPU.

Keywords: alexithymia, social interaction anxiousness, boredom proneness, problematic mobile phone use, college students

\section{Introduction}

Since the beginning of the 21 st century, information and communication technologies were developed rapidly. The use of a mobile phone has quickly increased globally. Simultaneously, these health consequences caused by using mobile phones anomalously have attracted to the public's attention. Problematic mobile phone use (PMPU) is defined as one's compulsive use of the mobile phone with the features of craving, dependence, tolerance, losing control, and increasing use to achieve satisfaction. ${ }^{1}$ The prevalence of PMPU has increased worldwide. For instance, based on the sample of Hong Kong adolescents, Leung observed that $27.4 \%$ adolescents were considered as PMPU. ${ }^{2}$ Then, Tavakolizadeh et al has reported $36.7 \%$ university students in their sample were defined as PMPU. ${ }^{3}$ In recent years, 
Wang and Zhang reported that $37.9 \%$ university students among a Chinese sample of 4000 university students were considered mobile phone addicts. ${ }^{4}$

On the one hand, the prevalence of PMPU has grown fast; on the other hand, the researchers found that PMPU can negatively affect multiple aspects of life. For example, it can cause physical and mental problems (eg, sleep disturbance and depression), ${ }^{5,6}$ increase cognition and social problems, ${ }^{7,8}$ and impede academic performance. ${ }^{9,10}$ Therefore, identifying the risk factors that might lead to PMPU and the internal mechanisms of the relationship between the risk factor and PMPU is of great significance.

Alexithymia is characterized by restrictions in the perception, differentiation and regulation of affects. It is considered to be an important vulnerability factor for the development of mental disorders. ${ }^{11,12}$ Moreover, previous studies showed that increased prevalence of alexithymia traits were found in patients with mental disorders, such as addictive disorders, ${ }^{13}$ anxiety disorders, ${ }^{14}$ and depression. ${ }^{15}$ Alexithymia is one of the risk factors that can lead to PMPU. Alexithymia refers to the one who has difficulties in identifying and describing the emotions of oneself and others and distinguishing feelings and bodily sensations and the externally oriented cognitive style. ${ }^{16}$ It has been demonstrated that alexithymia can cause PMPU in different samples of individuals. ${ }^{17-19}$ For example, previous studies reported that alexithymia is a critical predisposing factor for PMPU in Chinese college students. ${ }^{17,18}$ There is a relationship between alexithymia and PMPU. However, the internal mechanisms that account for this relationship are still unknown. Therefore, exploring the internal mechanism of this connection was a key issue in the research field of PMPU.

A potential explanation for the etiology of PMPU is the Interaction of Person-Affect-Cognition-Execution (I-PACE) model, which proposes that the addictive behavior is the result of an interaction between predisposing variables, affective and cognitive responses to specific stimuli and executive functions. ${ }^{20}$ In this model, the P-component refers to predisposing variables, which represent the core characteristics of people who are likely to be involved in the addiction process. ${ }^{21}$ Predisposing variables include general predisposing variables and behavior-specific predisposing variables. Alexithymia has a direct effect on PMPU and may influence PMPU through mediators such as cognitive factors and a person's characteristic variables. However, previous studies mainly pay attention to the cognitive factors that may explain the relationship between alexithymia and PMPU while neglecting the cognitive factors and personality variables. ${ }^{17,19}$ Therefore, this study aimed to investigate the influence of alexithymia on PMPU and the roles of potential cognitive factors and person's characteristic variables among college students. Based on previous studies, we proposed the first hypothesis of the present study: alexithymia can positively predict PMPU among college students (H1).

SIA is defined as excessive fear in social situations, where one believes that he or she will do something embarrassing or have anxiety symptoms (eg, blushing or sweating) that will be humiliating. ${ }^{22}$ SIA was often seen as roughly equivalent to social phobia, which was named social anxiety disorder (SAD). In the previous studies, these two terms were often used interchangeably. ${ }^{23} \mathrm{~A}$ recent meta-analysis proved that a small-to-moderate association $(r=0.22, p<0.001$, CI [0.17-0.28]) between problematic smartphone use and anxiety. ${ }^{24}$ Moreover, empirical studies have shown that SIA is a critical factor that influences individuals' PMPU. ${ }^{25,26}$

Meanwhile, previous studies found that SIA is positively correlated with alexithymia. ${ }^{27-29}$ For example, Dalbudak et al investigated the relationship between social interaction anxiety symptoms with alexithymia and university students' personality dimensions. ${ }^{28}$ They found a significantly positive correlation between alexithymia and social anxiety. Cheok and Proeve reported that SIA can be predicted by alexithymia. ${ }^{27}$ These results indicate that SIA may be an essential mediator (cognitive factor) that affects the relationship between alexithymia and PMPU. Based on the relevant studies and the above analysis, we proposed the second hypothesis of the current research: alexithymia can impact PMPU through the mediating role of SIA among college students (H2).

In contrast to boredom, BPS is a personality trait, in particular, an individual's disposition to experience boredom. ${ }^{30}$ Research has shown that BPS can predict PMPU. The pathway model of PMPU proposes that BPS is one of the extraversion pathways that account for PMPU. This pathway highlights that uncontrolled desires of using mobile phones are driving by the constant need for stimulation, such as driving by sensation-seeking. ${ }^{31}$ Previous studies also showed that BPS is closely associated with sensationseeking. ${ }^{32}$ This evidence indicates that people may seek novel stimuli to arouse themselves to the optimal level when they felt a high level of boredom in daily life. ${ }^{33}$ Due to the customization and multiple functions of mobile phones, in this case, boredom-prone people can obtain 
adequate stimulation across time and situations from the mobile phone.

Moreover, previous studies found that BPS is positively correlated with alexithymia. ${ }^{33,34}$ For example, Chen investigated the relationship between alexithymia symptoms with anxiety and depression in university students. ${ }^{34}$ They found a significantly positive correlation between alexithymia and BPS. Zhang et al reported that BPS could be predicted by alexithymia. ${ }^{33}$ These results indicate that BPS may be an important mediator (personality factor) that affect the relationship between alexithymia and PMPU. Based on the relevant studies and the above analysis, we proposed the third hypothesis of the current study: alexithymia can exert an impact on PMPU through the mediating role of BPS among college students (H3).

With the rapid development of technology applications, mobile phones allow individuals to escape face-to-face interactions or immediate responses and create a less intimate circumstance than real-life interactions. ${ }^{35}$ These functions of mobile phones provide adolescents with social anxiety another tool to interact with others. This may will lead individuals to escape from real-life interactions and immediate responses and increase the possibility of communicating with others via mobile phones. Moreover, a multitude of previous studies have found that the adolescents with social anxiety prefer online social interaction than face-to-face communication and were more inclined to use the mobile phone. ${ }^{36-38}$

Anxiety is considered as a kind of negative emotion. Previous studies have proven that individuals who felt boredom often experience negative affect, ${ }^{39}$ which indicated that boredom proneness correlates with anxious psychopathology. ${ }^{40-42}$ In fact, negative affect is conceptualized as a primary cause of experiencing boredom. ${ }^{43}$ Moreover, the directionality between negative affect (ie, anxiety) and boredom has been investigated with experiments and momentary ecological assessment. The results of the previous studies revealed that negative affect influences boredom. ${ }^{42,44}$ Furthermore, a lot of prior research has proven that BPS was correlated with PMPU. ${ }^{45,46}$ The above analysis showed that alexithymia may affect PMPU through the influence of SIA and BPS. In other words, SIA (cognitive factor) and BPS (personality factor) may act as serial mediators in the relationship between alexithymia and PMPU. Consequently, we proposed the last hypothesis of this study: alexithymia can exert an impact on mobile phone use through serial mediation of SIA and BPS among college students (H4).

\section{The Current Study}

The primary purpose of this study can be expressed in two aspects. First, the current research explores the internal mechanisms of the relationship between alexithymia and PMPU and provides a scientific basis for preventing PMPU among college students. Second, based on the analysis and the pieces of evidence from the previous studies, we plan to examine whether SIA (cognitive factor) and BPS (personality factor) play parallel and multiple mediators between the relationship of alexithymia and PMPU. Based on the I-PACE model and the results of the existing empirical studies, we assumed that (a) alexithymia can positively predict PMPU; (b) SIA and BPS would mediate the link between alexithymia and PMPU in a parallel way; and (c) SIA and BPS would mediate the relationship between alexithymia and PMPU in a serial way. All assumptions are shown in Figure 1.

Many empirical studies have indicated that alexithymia can lead to PMPU. ${ }^{17-19}$ However, the internal mechanisms of this relationship are still unknown. The present study constructed a multiple mediation model to explore the internal mechanisms of this relationship by integrating separate models. From a statistical and practical view, a multiple mediation model can not only offer more advantages than a simple mediation model, ${ }^{47}$ but also can integrate multiple mediation variables, and enable to comprehensively examine the complex processes and mechanisms of how the independent variables exert an influence on the dependent variables.

\section{Methods}

\section{Participants}

One thousand and three hundred college students were recruited as volunteers from two universities that are local in the Fujian and Zhejiang province of China by convenience sampling and took part in the current study. They were asked to complete the Internet-based survey via QR code. A total of 1267 participants were valid, which

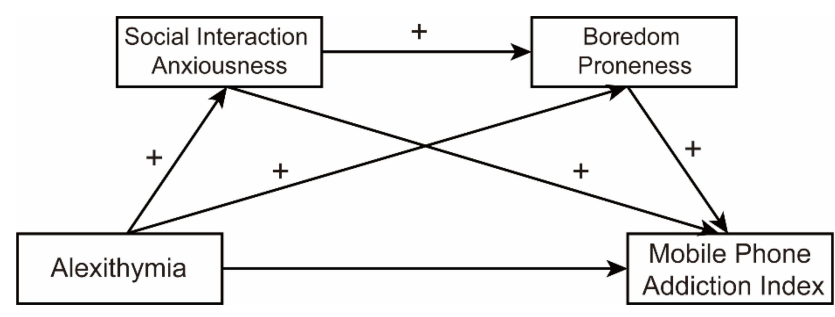

Figure I The multiple mediation model. 
accounted for $97.46 \%$ of the total sample. The college students who completed the survey below two standard deviations $(S D)$ of the average time were excluded $(\mathrm{N}=$ 33). The participants' age was ranged from 18 to 30 years, with a mean $(M)$ age of 20.36 years $(S D=0.97)$. Specifically, this study's final sample included 750 females (59.19\%) and 517 males (40.81\%). All participants reported that they have one constant Internet-accessible mobile phone. This study was authorized by the Ethics Committee of School of Education at Fujian Polytechnic Normal University, which was conducted per the Declaration of Helsinki. Participants who agreed to participate in the study gave their informed consent.

\section{Procedures}

The questionnaire's content and procedures were discussed with the psychological services departments of all participating universities before sampling. The participants were informed that their responses to questionnaires would be anonymous and confidential, and the data collected would be used for academic research. Then, the participants have received a QR code from their instructor and were asked to complete the survey via the QR code, which linked to the Chinese Star Survey website. This survey took approximately 20 minutes to complete all questionnaires. No incentives were provided to the participants.

\section{Measurements in This Study}

The PMPU was measured by the Mobile Phone Addiction Index (MPAI). ${ }^{2}$ This scale contains 17 items that assess the PMPU through the following four dimensions, withdrawal/escape, losing control and receiving complaints, anxiety, and craving, and loss of productivity. This scale was rated on a five-point scale, which one means not at all and five means always. The participants who reported higher scores on this scale indicate that there was a greater tendency of PMPU. Numbers of existing researches have demonstrated that the MPAI has good reliability and validity in Chinese youths and colleges. ${ }^{25,46}$ The Cronbach's $\alpha$ coefficient of the MPAI scale was 0.90 in the current study.

The Toronto Alexithymia-20 Scale (TAS-20) was used to evaluate the alexithymia. ${ }^{48}$ This scale is a self-report instrument with 20 items (ie, "I am often confused about my emotions"), which contains three aspects: (a) difficulties in describing feelings (DDF) (b) externally oriented thinking (EOT); and (c) difficulties in identifying feelings (DIF). TAS-20 was rated on a five-point scale, which one means strongly disagree and five means strongly agree. The higher score of the 20 items representing more severe alexithymia symptoms. TAS- 20 has been proven to be a reliable and valid instrument in Chinese populations. ${ }^{49}$ The Cronbach's $\alpha$ coefficient of the TAS- 20 scale was 0.86 in the current study.

SIA was assessed by the Social Interaction Anxiousness Scale (SIAS), which was developed by Leary and Kowalski. ${ }^{50}$ This scale is a 15 -item scale that evaluates the subjective anxiety and distress of the participants when they interact with different types of interactive partners (eg, strangers). In addition, it can also measure the subjective anxiety of people in distress in different social situations (ie, parties and job interviews). This scale is a seven-point Likert scale from "Not at all characteristic of me" to "Extremely characteristic of me", and the total scores are considered as the SIA index. A higher score means higher levels of subjective interaction anxiety. The SIAS-20 has been proven to be a reliable, effective instrument and has good validity and reliability in Chinese populations. ${ }^{51}$ The Cronbach's $\alpha$ coefficient was of this scale 0.87 in the current study.

The Boredom Proneness Scale short form (BPS-SF) was developed by Vodanovich et al in $2005 .{ }^{52}$ BPS-SF is a two-factor short form with a 12-item measure. The BPSSF is composed of two subscales: internal stimulation (6 items; eg, "I find it easy to entertain myself".) and external stimulation (6 items; eg, "Unless I am doing something exciting, even dangerous, I feel half dead and dull".). It is scored using a seven-point Likert scale. Response options range from " 1 = Highly disagree" to " $7=$ Highly agree". The scale is reliable and valid. ${ }^{29}$ In the current study, we used the scale's Chinese version and the Cronbach's $\alpha$ value for our sample was $0.78 .^{53}$

All statistical analysis was performed by SPSS software for Windows version 25.0 (SPSS, Inc., Chicago, IL) and the macro developed for the SPSS (ie, SPSS PROCESS macro).${ }^{54}$ First, descriptive statistics and correlational analyses were performed to descript and explained the relationship among the main variables (Hypothesis 1). After that, SIA (Hypothesis 2) or BPS (Hypothesis 3) as the potential mediator in the relationship between alexithymia and PMPU was tested by the Model 4 of SPSS PROCESS macro separately. ${ }^{54}$ Finally, Model 6 of the macro was performed to test the chain mediation effect of SIA and BPS (Hypothesis 4) analysis. It is worthy of knowing that the significance of the mediating effects was tested at $95 \%$ confidence intervals generated by 5000 
bootstrap samples and the statistical tests in this study were two-tailed with the alpha set at 0.05 .

\section{Result}

\section{Preliminary Analyses Common Method Bias}

Anonymous responses, reverse scores, and statistical analysis were applied to control the possible common method bias in the current study. In the data collection period, the anonymous responses and reverse scores method were performed. Besides, in the data analysis period, the Harman single factor method was conducted to test the common method bias. ${ }^{53,55}$ Harman's single factor test is one technique to identify common method variance. If a single factor emerges or one general factor will account for the majority of the covariance among the measures, then it is concluded that a substantial amount of common method variance is present. The results showed that eleven factors were obtained after the exploratory factor analysis, and the interpretation rate of the first factor $(24.20 \%)$ was lower than the threshold of $40 \%$, which suggested that there was no serious common method bias.

\section{Description and Correlation Statistics}

For the demographic variables, such as gender and age, we did not observe positively or negatively associated with PMPU (the outcome variable). Hence, the demographic variables were excluded in the following analyses.

As expected, we found that alexithymia was positively associated with SIA, BPS, and PMPU. Also, SIA was positively associated with alexithymia, PMPU, and BPS. Besides, the BPS was positively associated with alexithymia, SIA, and PMPU. Table 1 shows the description indexes (ie, $M$ and $S D$ ) and the correlation results of the main variables.

\section{Testing the Mediation Role of Social Interaction Anxiousness}

Testing the mediation role of SIA (Hypothesis 2) between alexithymia and PMPU was performed via the PROCESS macro with Model 4. The results showed that alexithymia was positively related to PMPU $(\beta=0.39, p$ $<0.001)$ and SIA $(\beta=0.60, p<0.001)$. Moreover, both alexithymia $(\beta=0.39, p<0.001)$ and SIA $(\beta=0.33, p$ $<0.001$ ) were positively related to PMPU. Furthermore, the result of bias-corrected percentile bootstrap analysis revealed that the mediator of SIA accounted for $33.86 \%$ of the total effect on the relationship between alexithymia and PMPU was significant $(\mathrm{ab}=0.20, S E=0.02$, $95 \% \mathrm{CI}=[0.15,0.24])$. More details can be seen in Table 2. Therefore, social interaction anxiousness partially mediated the relationship between alexithymia and problematic mobile phone use, which supporting Hypothesis 2.

\section{Testing the Mediation Role of BPS}

Testing the mediation role of BPS (Hypothesis 3) between alexithymia and PMPU was performed via the PROCESS macro with Model 4 . The results showed that alexithymia was positively related to PMPU and BPS ( $\beta_{\text {alexithymia }}=$ $\left.0.38, p<0.001 ; \beta_{B P S}=0.87, p<0.001\right)$. Moreover, both alexithymia $(\beta=0.38, p<0.001)$ and $\operatorname{BPS}(\beta=0.34, p<$ 0.001 ) were positively related to PMPU. Furthermore, the result of bias-corrected percentile bootstrap analysis revealed that BPS had a significant mediation effect on the relationship between alexithymia and PMPU $(\mathrm{ab}=$ $0.21, \mathrm{SE}=0.03,95 \% \mathrm{CI}=[0.15,0.27])$, which accounted for $35.55 \%$ of the total effect (more details can be seen in Table 3). Therefore, BPS partially mediated the relationship between alexithymia and PMPU, which supports Hypothesis 3.

Table I Descriptive Statistics and Inter-Correlations Between the Main Variables $(\mathrm{N}=1267)$

\begin{tabular}{|l|c|c|c|c|c|c|c|c|}
\hline & M & SD & I & $\mathbf{2}$ & $\mathbf{3}$ & $\mathbf{4}$ & $\mathbf{5}$ & $\mathbf{6}$ \\
\hline I. Gender & 0.59 & 0.49 & $\mathrm{I}$ & - & - & - & - & - \\
2. Age & 20.36 & 0.97 & $-0.10^{* *}$ & $\mathrm{I}$ & - & - & - \\
3. TAS & 2.61 & 0.50 & -0.00 & -0.02 & $\mathrm{I}$ & - & - \\
4. MPAI & 2.78 & 0.72 & 0.05 & -0.06 & $0.40^{* *}$ & $\mathrm{I}$ & - & - \\
5. SIA & 3.12 & 0.62 & $0.18^{* *}$ & $-0.06^{*}$ & $0.48^{* *}$ & $0.41^{* *}$ & - \\
6. BPS & 3.49 & 0.73 & $-0.09 * *$ & $-0.08^{* *}$ & $0.59^{* *}$ & $0.40^{* *}$ & $0.43^{* *}$ & - \\
\hline
\end{tabular}

Note: $*_{p}<0.05 ; * *_{p}<0.01$.

Abbreviations: M, mean; SD, standard deviation, Gender was dummy coded, which zero stands for male, and one stands for female; TAS, alexithymia; MPAl, mobile phone addiction index, which was used to measure the problematic mobile phone use (PMPU); SIA, social interaction anxiousness; BPS, boredom proneness. 
Table 2 Testing Social Interaction Anxiousness as a Mediator in the Relationship Between Alexithymia and Problematic Mobile Phone Use $(\mathrm{N}=1267)$

\begin{tabular}{|l|c|c|c|c|c|c|c|c|}
\hline Criterion & Predictors & $\boldsymbol{R}$ & $\boldsymbol{R}^{\mathbf{2}}$ & $\boldsymbol{F}$ & $\boldsymbol{\beta}$ & Boot LLCl & Boot ULCI & $\boldsymbol{t}$ \\
\hline SIA & TAS & 0.48 & 0.23 & $375.65 * * *$ & 0.60 & 0.54 & 0.66 & $19.38 * * *$ \\
\hline MPAI & TAS & 0.48 & 0.23 & $184.23 * * *$ & 0.39 & 0.31 & 0.47 & $9.47 * * *$ \\
& SIA & & & & 0.33 & 0.27 & 0.39 & $10.13 * * *$ \\
\hline
\end{tabular}

Notes: ${ }^{* * *} p<0.001$. Standardized regression coefficients were reported. Bootstrap sample size $=1000$.

Abbreviations: TAS, alexithymia; MPAI, mobile phone addiction index, which was used to measure the problematic mobile phone use (PMPU); SIA, social interaction anxiousness; LL, low limit; UL, upper limit; Cl, confidence interval.

Table 3 Testing Boredom Proneness as a Mediator in the Relationship Between Alexithymia and Problematic Mobile Phone Use $(\mathrm{N}=1267)$

\begin{tabular}{|l|c|c|c|c|c|c|c|c|}
\hline Criterion & Predictors & $\boldsymbol{R}$ & $\boldsymbol{R}^{\mathbf{2}}$ & $\boldsymbol{F}$ & $\boldsymbol{\beta}$ & Boot LLCl & Boot ULCl & $\boldsymbol{t}$ \\
\hline BPS & TAS & 0.59 & 0.35 & $690.98 * * *$ & 0.87 & 0.78 & 0.93 & $26.27 * * *$ \\
\hline MPAI & TAS & 0.45 & 0.20 & $158.53 * * *$ & 0.38 & 0.29 & 0.47 & $9.47 * * *$ \\
& BPS & & & & 0.24 & 0.18 & 0.30 & $7.72 * * *$ \\
\hline
\end{tabular}

Notes: Standardized regression coefficients were reported. Bootstrap sample size $=1000 . * * * p<0.001$.

Abbreviations: TAS, alexithymia; MPAl, mobile phone addiction index, which was used to measure the problematic mobile phone use (PMPU); BPS, boredom proneness; LL, low limit; UL, upper limit; $\mathrm{Cl}$, confidence interval.

\section{Testing the Multiple Mediation Model}

We utilized Model 6 of PROCESS to examine the multiple mediation model (Hypothesis 4). ${ }^{54}$ The results showed that all pathway coefficients were significant, more details can be seen in Table 4 and Figure 2. More specifically, the pathways for "TAS $\rightarrow$ SIA $\rightarrow$ MPAI" (indirect effect $=0.18$, $95 \% \mathrm{CI}=[0.13,0.22]$ ), and "TAS $\rightarrow \mathrm{BPS} \rightarrow$ MPAI" (indirect effect $=0.14,95 \% \mathrm{CI}=[0.08,0.19])$ were significant, which suggested that SIA and BPS mediated the association between alexithymia and PMPU. Similarly, the sequential pathway for "TAS $\rightarrow$ SIA $\rightarrow$ BPS $\rightarrow$ MPAI" was significant (indirect effect $=0.02,95 \% \mathrm{CI}=[0.01$, $0.03]$ ), implying that alexithymia was serially associated with higher SIA $(\beta=0.60, p<0.01)$, higher BPS $(\beta=$ $0.21, p<0.01)$, as well as PMPU $(\beta=0.19, p<0.01)$.

By the same token, the residual direct pathway for "TAS $\rightarrow$ MPAI" was also significant $(\beta=0.25, p<$ $0.01)$. Thus, SIA and BPS mediated the relation between alexithymia and PMPU, not only in parallel way but also sequentially way. Summarily, this multiple mediation model accounted for a significant amount of variance in college students' PMPU, which supporting Hypothesis 4.

Table 4 Testing the Pathways of the Multiple Mediation Model $(\mathrm{N}=1267)$

\begin{tabular}{|l|c|c|c|c|}
\hline Effect & $\boldsymbol{\beta}$ & SE & 95\% Boot LLCI & 95\% Boot ULCI \\
\hline Direct effects & & & & 0.54 \\
TAS $\rightarrow$ SIA & $0.60^{* * *}$ & 0.03 & 0.67 & 0.66 \\
TAS $\rightarrow$ BPS & $0.74^{* * *}$ & 0.04 & 0.16 & 0.81 \\
SIA $\rightarrow$ BPS & $0.21^{* * *}$ & 0.03 & 0.16 & 0.27 \\
TAS $\rightarrow$ MPAI & $0.25 * * *$ & 0.05 & 0.23 & 0.36 \\
SIA $\rightarrow$ MPAI & $0.29 * * *$ & 0.03 & 0.13 & 0.25 \\
BPS $\rightarrow$ MPAI & $0.19 * * *$ & 0.03 & & \\
\hline Indirect effects & & & 0.13 & \\
TAS $\rightarrow$ SIA $\rightarrow$ MPAI & 0.18 & 0.02 & 0.08 & 0.22 \\
TAS $\rightarrow$ BPS $\rightarrow$ MPAI & 0.14 & 0.03 & 0.01 & 0.19 \\
TAS $\rightarrow$ SIA $\rightarrow$ BPS $\rightarrow$ MPAI & 0.02 & 0.01 & & \\
\hline
\end{tabular}

Notes: Standardized regression coefficients were reported. Bootstrap sample size $=1000 . * * * p<0.001$.

Abbreviations: TAS, alexithymia; MPAI, mobile phone addiction index, which was used to measure the problematic mobile phone use (PMPU); SIA, social interaction anxiousness; BPS, boredom proneness; LL, low limit; UL, upper limit; Cl, confidence interval. 


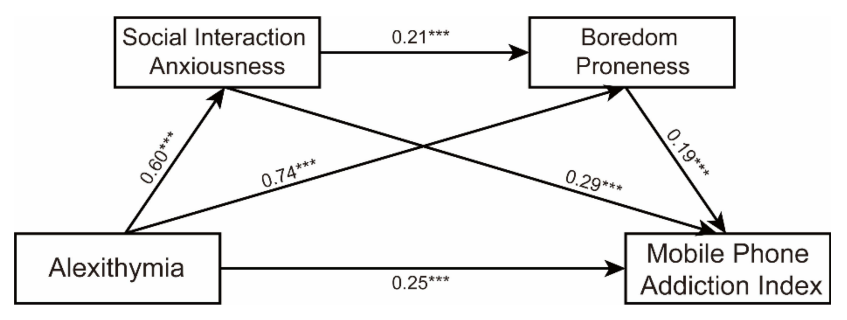

Figure 2 The pathway coefficients of multiple mediation model. *** $p<0.00$ I.

\section{Discussion}

The current research aims at gaining a deeper understanding of the internal mechanism between alexithymia and PMPU. Based on the theoretical and empirical analysis, we examined the multiple mediating roles of SIA and BPS (parallel and serial) in the relationship between alexithymia and PMPU among university students. The hypothesized multiple mediators model (SIA and BPS) was supported by the results. This result highlights the important role of SIA and BPS in the impact path of alexithymia and PMPU. In addition, this result also offers valuable insights into prevention and intervention. More specifically, in the current study, a positive correlation between alexithymia and PMPU was observed, and a significant positive predictive role of alexithymia to PMPU was also detected. These findings are in line with previous studies. ${ }^{17-19}$ It can be reasoned that alexithymia is a vital risk factor for PMPU. Therefore, the first hypothesis of this study was validated.

\section{SIA as a Mediator}

SIA played a mediating role in the relationship between alexithymia and PMPU, which is indicated by the results. Hence, the hypothesis $\mathrm{H} 2$ was validated. SIA refers to the tendency to fear and avoid social interactions due to concern about being negatively evaluated, rejected or embarrassed. ${ }^{56}$ Alexithymia is considered to be a stable personality trait and has been linked to psychological symptoms, including social interaction anxiety. ${ }^{29,57}$ Individuals with alexithymia could not meet regular social needs due to their inability to identify, describe, and communicate feelings to others, which may lead to interaction anxiety. Accordingly, they would reduce interaction anxiety by adopting other forms of social interaction to compensate for their unmet needs in real life. Mobile phone, as the most common social tool, could meet their social needs in the virtual network. Therefore, individuals are more likely to have problematic phone use if they have a high level of alexithymia symptoms.

\section{BPS as a Mediator}

The findings of the current study indicated that college students with high levels of alexithymia symptoms tend to experience boredom that subsequently leads to PMPU, which suggested that BPS also played a mediating role in the relationship between alexithymia and PMPU. Therefore, the hypothesis H3 was validated. The result showed that alexithymia was positively associated with boredom proneness. This result was in line with prior research documenting the mediating role of boredom proneness in the relationship between unfavorable factors (ie, alexithymia) and individual maladaptive behaviors (ie, PMPU). ${ }^{33,58,59}$ Moreover, the I-PACE model emphasizes that addictive behavior is the result of an interaction between predisposing variables, affective and cognitive responses to specific stimuli and executive functions. ${ }^{20}$ Hence, the college students with a high score of alexithymia are susceptible to feel bored, and finally, lead to the PMPU.

In addition, we observed that boredom proneness was positively associated with PMPU. This finding was consistent with the Compensatory Internet Use Theory, ${ }^{60}$ which hypothesizes that the individuals in distressing conditions (ie, interpersonal interaction dilemma) have a strong motivation to compensate for their psychosocial problems via the internet. Consequently, college students with the alexithymia symptoms tend to use mobile phone as a kind of social interaction strategy to evade from boredom experience due to that they could not identify and describe the feelings of others when they interacted with others. Their over-reliance on mobile phone might in turn lead to PMPU.

\section{SIA and BPS as Mediators}

More importantly, we also observed that alexithymia can exert an impact on PMPU through the serial mediation of SIA and BPS among college students. Therefore, the final hypothesis of the current study was validated. These findings support the viewpoint of the I-PACE model, which asserts that addictive behavior is the result of an interaction between predisposing variables, affective and cognitive responses to specific stimuli and executive functions. ${ }^{15}$ According to the I-PACE model (personal factors), personality traits, biological variables, and psychopathology, which may influence excessive mobile phone use. $^{53,61,62}$ In the current study, we found that those who have difficulties in identifying and describing 
the emotions of oneself and others were increasing the level of SIA and BPS and finally led to the PMPU, which was in line with the I-PACE model. Moreover, these negative cognitive and emotional experiences may enhance the avoidance of the individuals' real-life communication, thereby finally increasing the possibility to use the mobile phone problematic when they try to interact with others via the internet.

This study, from a cognitive and personality perspective, provides an in-depth elucidation of the specific internal psychological mechanisms about how alexithymia accounts for PMPU. When university students have alexithymia symptoms, such as they have difficulties in identifying and describing the emotions of oneself and others to some degree, the quality and efficiency of social interaction of these students would tend to a low level and make them anxious. A lot of anxiety due to the low level of social interaction makes individuals feel bored interacting with others and making the individuals seek the novel stimulus. These reactions to the personal factors are important in I-PACE, and include risk or buffering paths to excessive mobile phone use, such as cognitive bias, coping, and disinhibition. ${ }^{21,63}$ Such response/reaction variables can exacerbate or lessen how personal factors influence mobile phone overuse.

\section{Implications for Practice}

The findings of the current study have vital implications for practice. First, given that BPS is a critical factor that explains how alexithymia exerts an impact on PMPU. It seems that reducing the BPS of the individuals may reduce the PMPU not only for the people with alexithymia symptoms but also for the individuals without alexithymia. Hence, the practitioners can develop some courses or activities with novel stimuli to help the boredom-prone people to arouse themselves to the optimal level. Second, given that SIA is another critical factor that explains how alexithymia exerts an impact on PMPU. It seems that reducing the social interaction anxiety of the students will be a useful way to reduce the PMPU. Therefore, the school curriculum can aim at teaching some social interaction skills to college students, and the practitioners can provide some face-to-face interactions for the individuals to practice the skills that learned from the course. Generally, the current study provides guidance to practitioners who seek to identify potential leverage points in the prevention of PMPU.

\section{Limitations and Future Directions}

In summary, this study examines the multiple mediating roles of SIA and BPS and provides a deeper understanding of the internal mechanism between alexithymia and PMPU. However, some limitations should be noted. First, selfreported method was applied to collect the data points, which may cause recall bias. Although the result showed that no common method biases were observed in the current study. Hence, it is a need to collect data to replicate the results from multiple sources. Second, the cross-sectional design was used in this study. Although we have controlled the covariates in the analyses and did some supplementary analyses to improve the explanatory power, owing to the non-experimental design, it was impossible to draw causal conclusions. The current results should be validated with the experimental design in the future. Third, emotional intelligence (EI) is the ability to reason about emotions and utilize affect-laden information to guide one's thinking as well as actions. EI is widely regarded as an important predictor of a diverse set of health and life success outcomes. There is growing evidence to demonstrate the role that EI plays in the development of both substance use and behavioral addictions. ${ }^{64}$ EI has been found to have both a direct effect on gaming addiction severity and indirect effect via escape motivation - a form of avoidance coping. ${ }^{65,66}$ The above studies indicated that EI plays in the development of both substance use and behavioral addictions. What is the role of EI between alexithymia and PMPU is needed to explore in the future. Finally, since these data were collected within university students, the applicability of the results should be validated with diverse populations in the future.

\section{Conclusion}

In summary, based on the Interaction of Person-AffectCognition-Execution model, this study examines the multiple mediating roles of SIA and BPS and aim at providing a deeper understanding of the internal mechanism between alexithymia and PMPU. We constructed a multiple mediation model to explore the internal mechanisms of this relationship by integrating separate models. The findings indicated that Alexithymia was positively linked with PMPU; (2) both SIA and BPS mediated the link between alexithymia and PMPU; and (3) a serial indirect pathway emerged (ie, Alexithymia $\rightarrow$ SIA $\rightarrow$ BPS $\rightarrow$ PMPU). In other words, the alexithymia symptoms not only can influence PMPU in a simple indirect way (parallel mediation) by simultaneously increasing social interaction anxiousness and boredom proneness but also in a 
complex indirect way (serial mediation) by increasing the level of boredom proneness through increasing social interaction anxiousness. Besides, these findings provide some insights into the prevention and intervention of problematic mobile phone use. More specifically, the practitioners can develop some courses or activities with novel stimuli to help the boredom-prone people to arouse themselves to the optimal level or the designer of the school curriculum can develop some social interaction skills classes to college students for the individuals to practice the skills that learned from the course.

\section{Data Sharing Statement}

The author has specified no data sets for the following reason: The data that has been used is confidential. Due to the sensitive nature of the questions asked in this study, survey respondents were assured raw data would remain confidential and would not be shared.

\section{Ethics Statement and Informed Consent}

This study was conducted in accordance with the Declaration of Helsinki. The Ethics Committee of School of Education at Fujian Polytechnic Normal University approved the study. This study was also approved by parents, school authorities, and principals. The participants interested in participating read the informed consent which contained the objectives, benefits, and risks of participation; those who agreed to participate in the study gave their informed consent, accepting to continue with the survey. The participants were informed that their responses to questionnaires would be anonymous and confidential, and the data collected would be used for academic research only.

\section{Acknowledgments}

Thanks to the participants who participated in this study. We are very grateful to the editor and reviewers for their work and their suggestions for this paper. We would like to thank our colleagues at ZJNU and FPNU. Weilong Xiao and Hui Zhou should be considered co-first authors.

\section{Author Contributions}

All authors made a significant contribution to the work reported, whether that is in the conception, study design, execution, acquisition of data, analysis and interpretation, or in all these areas; took part in drafting or critically reviewing the article; gave final approval of the version to be published; have agreed on the journal to which the article has been submitted; and agree to be accountable for all aspects of the work. It is important to note that Hui Zhou made an outstanding contribution in revising the manuscript, especially for the revision of reviewer 2's comments.

\section{Funding}

This work was funded by the key project of the Ministry of Education of the National Educational Science Planning (DHA210341).

\section{Disclosure}

The authors declare no competing interests in this work.

\section{References}

1. Lopez-Fernandez O. Short version of the Smartphone Addiction Scale adapted to Spanish and French: towards a cross-cultural research in problematic mobile phone use. Addict Behav. 2017;64:275-280. doi:10.1016/j.addbeh.2015.11.013

2. Leung L. Linking psychological attributes to addiction and improper use of the mobile phone among adolescents in Hong Kong. J Child Media. 2008;2(2):93-113. doi:10.1080/17482790802078565

3. Tavakolizadeh J, Atarodi A, Ahmadpour S, et al. The prevalence of excessive mobile phone use and its relation with mental health status and demographic factors among the students of Gonabad University of Medical Sciences in 2011-2012. Razavi Int J Med. 2014;2(1): e15527. doi:10.5812/rijm.15527

4. Wang YQ, Zhang Y. Relation of mobile phone addiction to perceived social support and subjective well-being in adolescent students. Chin Ment Health J. 2015;29(11):868-873.

5. Zhang MX, Wu A. Effects of smartphone addiction on sleep quality among Chinese university students: the mediating role of self-regulation and bedtime procrastination. Addict Behav. 2020;111:106552. doi:10.1016/j.addbeh.2020.106552

6. Sohn S, Rees P, Wildridge B, Kalk NJ, Carter B. Prevalence of problematic smartphone usage and associated mental health outcomes amongst children and young people: a systematic review, meta-analysis and GRADE of the evidence. BMC Psychiatr. 2019;19(1):1-10.

7. Chen L, Yan Z, Tang W, Yang F, Xie X, He J. Mobile phone addiction levels and negative emotions among Chinese young adults: the mediating role of interpersonal problems. Comput Human Behav. 2016;55:856-866. doi:10.1016/j.chb.2015.10.030

8. Hong W, Liu RD, Ding Y, Sheng X, Zhen R. Mobile phone addiction and cognitive failures in daily life: the mediating roles of sleep duration and quality and the moderating role of trait self-regulation. Addict Behav. 2020;107:106383. doi:10.1016/j.addbeh.2020.106383

9. Samaha M, Hawi NS. Relationships among smartphone addiction, stress, academic performance, and satisfaction with life. Comput Human Behav. 2016;57:321-325. doi:10.1016/j.chb.2015.12.045

10. Lepp A, Barkley JE, Karpinski AC. The relationship between cell phone use and academic performance in a sample of US adolescent students. Sage Open. 2015;5(1):2158244015573169. doi:10.1177/ 2158244015573169

11. Leweke F, Leichsenring F, Kruse J, Hermes S. Is alexithymia associated with specific mental disorders. Psychopathology. 2012;45 (1):22-28. doi:10.1159/000325170 
12. Zakiei A, Karami J. Relationship of alexithymia, positive affect and negative affect with mental disorders. Med J Tabriz Univ Med Sci. 2013;35(4):38-45.

13. Haviland MG, Hendryx MS, Shaw DG, Henry JP. Alexithymia in women and men hospitalized for psychoactive substance dependence. Compr Psychiatry. 1994;35(2):124-128. doi:10.1016/0010-440X(94) 90056-N

14. Cox BJ, Swinson RP, Shulman ID, Bourdeau D. Alexithymia in panic disorder and social phobia. Compr Psychiatry. 1995;36(3):195-198. doi:10.1016/0010-440X(95)90081-6

15. Honkalampi K, Hintikka J, Antikainen R, Lehtonen J, Viinamäki H. Alexithymia in patients with major depressive disorder and comorbid cluster C personality disorders: a 6-month follow-up study. J Pers Disord. 2001;15(3):245-254. doi:10.1521/pedi.15.3.245.19211

16. Taylor GJ, Bagby RM, Parker JD. Disorders of Affect Regulation: Alexithymia in Medical and Psychiatric Illness. Cambridge University Press; 1999.

17. Hao Z, Jin L. Alexithymia and problematic mobile phone use: a moderated mediation model. Front Psychol. 2020;11:541507. doi:10.3389/fpsyg.2020.541507

18. Mei S, Xu G, Gao T, Ren H, Li J. The relationship between adolescent students' alexithymia and mobile phone addiction: testing mediation and moderation effects. BMC Psychiatr. 2018;18(1):1-7. doi:10.1186/s12888-018-1891-8

19. Hao Z, Jin L, Li Y, Huang J. Alexithymia and mobile phone addiction in Chinese undergraduate students: the roles of mobile phone use patterns. Comput Human Behav. 2019;97:51-59. doi:10.1016/j.chb.2019.03.001

20. Brand M, Wegmann E, Stark R, et al. The Interaction of Person-AffectCognition-Execution (I-PACE) model for addictive behaviors: update, generalization to addictive behaviors beyond internet-use disorders, and specification of the process character of addictive behaviors. Neurosci Biobehav Rev. 2019;104:1-10. doi:10.1016/j.neubiorev.2019.06.032

21. Brand M, Young KS, Laier C, Wolfling K, Potenza MN. Integrating psychological and neurobiological considerations regarding the development and maintenance of specific internet-use disorders: an interaction of person-affect-cognition execution (I-PACE) model. Neurosci Biobehav Rev. 2016;71:252-266. doi:10.1016/j.neubiorev.2016.08.033

22. American Psychiatric Association. Diagnostic and Statistical Manual of Mental Disorders. 5th ed. Arlington, VA: American Psychiatric Association; 2013. Text Revision.

23. Alkis Y, Kadirhan Z, Sat M. Development and validation of social anxiety scale for social media users. Comput Human Behav. 2017;72:296-303. doi:10.1016/j.chb.2017.03.011

24. Vahedi Z, Saiphoo A. The association between smartphone use, stress, and anxiety: a meta-analytic review. Stress Health. 2018;34 (3):347-358. doi:10.1002/smi.2805

25. Liu QQ, Yang XJ, Hu YT, Zhang CY, Nie YG. How and when is family dysfunction associated with adolescent mobile phone addiction? Testing a moderated mediation model. Child Youth Serv Rev. 2020;111:104827. doi:10.1016/j.childyouth.2020.104827

26. You Z, Zhang Y, Zhang L, Xu Y, Chen X. How does self-esteem affect mobile phone addiction? The mediating role of social anxiety and interpersonal sensitivity. Psychiatry Res. 2019;271:526-531. doi:10.1016/j.psychres.2018.12.040

27. Cheok F, Proeve M. The role of perspective taking and alexithymia in associations between shame, guilt, and social anxiety. J Cogn Psychother. 2019;33(4):286-300. doi:10.1891/0889-8391.33.4.286

28. Dalbudak E, Evren C, Aldemir S, Coskun KS, Ugurlu H, Ugurlu H. Alexithymia and personality in relation to social anxiety among university students. Psychiatry Res. 2013;209(2):167-172. doi:10.1016/j. psychres.2012.11.027

29. Kashdan TB, Farmer AS. Differentiating emotions across contexts: comparing adults with and without social anxiety disorder using random, social interaction, and daily experience sampling. Emotion. 2014;14(3):629-638. doi:10.1037/a0035796
30. Sung B, Lee S, Teow T. Revalidating the boredom proneness scales short from (BPS-SF). Pers Individ Dif. 2021;168:110364. doi:10.1016/j.paid.2020.110364

31. Billieux J, Maurage P, Lopez-Fernandez O, Kuss DJ, Griffiths MD. Can disordered mobile phone use be considered a behavioral addiction? An update on current evidence and a comprehensive model for future research. Curr Addict Rep. 2015;2(2):156-162. doi:10.1007/ s40429-015-0054-y

32. Hwang H, Park S. Sensation seeking and smoking behaviors among adolescents in the Republic of Korea. Addict Behav. 2015;45:239244. doi:10.1016/j.addbeh.2015.01.041

33. Zhang Y, Li S, Yu G. Alexithymia and aggression among adolescents: the mediating role of boredom proneness. Chin J Clin Psychol. 2020;28(2):383-386+399.

34. Chen H. The relationship between alexithymia and emotional symptoms: mediating effects of boredom proneness. Chin J Clin Psychol. 2016;24(04):648-651.

35. Lee YK, Chang CT, Lin Y, Cheng ZH. The dark side of smartphone usage: psychological traits, compulsive behavior and technostress. Comput Human Behav. 2014;31:373-383. doi:10.1016/j.chb.2013.10.047

36. Sapacz M, Rockman G, Clark J. Are we addicted to our cell phones? Comput Human Behav. 2016;57:153-159. doi:10.1016/j. chb.2015.12.004

37. Fioravanti G, Dèttore D, Casale S. Adolescent internet addiction: testing the association between self-esteem, the perception of internet attributes, and preference for online social interactions. Cyberpsychol Behav Soc Netw. 2012;15(6):318-323. doi:10.1089/cyber.2011.0358

38. Caplan SE. Relations among loneliness, social anxiety, and problematic internet use. Cyber Psychol Behav. 2006;10(2):234-242. doi:10.1089/cpb.2006.9963

39. Struk AA, Carriere JS, Cheyne JA, Danckert J. A short boredom proneness scale: development and psychometric properties. Assessment. 2017;24(3):346-359. doi:10.1177/1073191115609996

40. Elhai JD, Vasquez JK, Lustgarten SD, Levine JC, Hall BJ. Proneness to boredom mediates relationships between problematic smartphone use with depression and anxiety severity. Soc Sci Comput Rev. 2018;36(6):707-720. doi:10.1177/0894439317741087

41. Goldberg YK, Eastwood JD, Laguardia J, Danckert J. Boredom: an emotional experience distinct from apathy, anhedonia, or depression. $J$ Soc Clin Psychol. 2011;30(6):647-666. doi:10.1521/ jscp.2011.30.6.647

42. Sommers J, Vodanovich SJ. Boredom proneness: its relationship to psychological- and physical-health symptoms. $J$ Clin Psychol. 2000;56(1):149-155. 56:1<149::AID-JCLP14>3.0.CO;2-Y

43. Eastwood JD, Frischen A, Fenske MJ, Smilek D. The unengaged mind: defining boredom in terms of attention. Perspect Psychol Sci. 2012;7(5):482-495. doi:10.1177/1745691612456044

44. Mercer K, Eastwood J. Is boredom associated with problem gambling behaviour? It depends on what you mean by 'boredom'. Int Gambl Stud. 2010;10:91-104. doi:10.1080/14459791003754414

45. Wang Y, Yang H, Montag C, Elhai JD. Boredom proneness and rumination mediate relationships between depression and anxiety with problematic smartphone use severity. Curr Psychol. 2020:111. doi:10.1007/s12144-020-01052-0

46. Yang XJ, Liu QQ, Lian SL, Zhou ZK. Are bored minds more likely to be addicted? The relationship between boredom proneness and problematic mobile phone use. Addict Behav. 2020;108:106426. doi:10.1016/j.addbeh.2020.106426

47. Preacher KJ, Hayes AF. Asymptotic and resampling strategies for assessing and comparing indirect effects in multiple mediator models. Behav Res Methods. 2008;40(3):879-891. doi:10.3758/BRM.40.3.879

48. Bagby RM, Parker JD, Taylor GJ. The twenty-item Toronto Alexithymia Scale-I. Item selection and cross-validation of the factor structure. J Psychosom Res. 1994;38(1):23-32. doi:10.1016/00223999(94)90005-1 
49. Tang W, Hu T, Yang L, Xu J. The role of alexithymia in the mental health problems of home-quarantined university students during the COVID-19 pandemic in China. Pers Individ Dif. 2020;165:110131. doi:10.1016/j.paid.2020.110131

50. Leary MR, Kowalski RM. The interaction anxiousness scale: construct and criterion-related validity. J Pers Assess. 1993;61(1):136146. doi:10.1207/s15327752jpa6101_10

51. Cao J, Yang J, Zhou Y, et al. The effect of Interaction Anxiousness Scale and Brief Social Phobia Scale for screening social anxiety disorder in adolescent students: a study on discriminative validity. $J$ Ment Health. 2016;25(6):500-505. doi:10.3109/ 09638237.2015.1124391

52. Vodanovich SJ, Wallace JC, Kass SJ. A confirmatory approach to the factor structure of the Boredom Proneness Scale: evidence for a twofactor short form. J Pers Assess. 2005;85(3):295-303. doi:10.1207/ s15327752jpa8503_05

53. Peng J, Guo W, Zhao L, Han X, Wu S. Short boredom proneness scale: adaptation and validation of a Chinese version with college students. Soc Behav Personal. 2020;48(2):1-8.

54. Hayes AF. Introduction to Mediation, Moderation, and Conditional Process Analysis: A Regression-Based Approach. New York: Guilford Press; 2013.

55. Podsakoff PM, MacKenzie SB, Lee JY, Podsakoff NP. Common method biases in behavioral research: a critical review of the literature and recommended remedies. J Appl Psychol. 2003;88(5):879903. doi:10.1037/0021-9010.88.5.879

56. Merikangas KR, Avenevoli S, Acharyya S, Zhang H, Angst J. The spectrum of social phobia in the Zurich cohort study of young adults. Biol Psychiatry. 2002;51(1):81-91. doi:10.1016/S0006-3223(01) 01309-9

57. Mennin DS, McLaughlin KA, Flanagan TJ. Emotion regulation deficits in generalized anxiety disorder, social anxiety disorder, and their co-occurrence. J Anxiety Disord. 2009;23(7):866-871. doi:10.1016/j. janxdis.2009.04.006
58. Yan L, Gan Y, Ding X, Wu J, Duan H. The relationship between perceived stress and emotional distress during the COVID-19 outbreak: effects of boredom proneness and coping style. J Anxiety Disord. 2020;77:102328. doi:10.1016/j.janxdis.2020.102328

59. Holte AJ, Ferraro FR. Anxious, bored, and (maybe) missing out: evaluation of anxiety attachment, boredom proneness, and fear of missing out (FoMO). Comput Human Behav. 2020;112:106465. doi:10.1016/j.chb.2020.106465

60. Kardefelt-Winther D. A conceptual and methodological critique of internet addiction research: towards a model of compensatory internet use. Comput Human Behav. 2014;31:351-354. doi:10.1016/j. chb.2013.10.059

61. Lachmann B, Duke É, Sariyska R, Montag C. Who's addicted to the smartphone and/or the internet? Psychol Popular Media Culture. 2019;8(3):182-189. doi:10.1037/ppm0000172

62. Peterka-Bonetta J, Sindermann C, Elhai JD, Montag C. Personality associations with smartphone and internet use disorder: a comparison study including links to impulsivity and social anxiety. Front Public Health. 2019;7. doi:10.3389/fpubh.2019.00127

63. Wegmann E, Oberst U, Stodt B, Brand M. Online-specific fear of missing out and internet-use expectancies contribute to symptoms of internet-communication disorder. Addict Behav Rep. 2017;5:33-42. doi:10.1016/j.abrep.2017.04.001

64. Henning C, Crane AG, Taylor RN, Parker JD. Emotional intelligence: relevance and implications for addiction. Curr Addict Rep. 2021;8:1-7.

65. Mascia ML, Agus M, Penna MP. Emotional intelligence, self-regulation, smartphone addiction: which relationship with student wellbeing and quality of life? Front Psychol. 2020;11:375. doi:10.3389/ fpsyg.2020.00375

66. Sun J, Liu Q, Yu S. Child neglect, psychological abuse and smartphone addiction among Chinese adolescents: the roles of emotional intelligence and coping style. Comput Human Behav. 2019;90:74-83. doi:10.1016/j.chb.2018.08.032
Psychology Research and Behavior Management

\section{Publish your work in this journal}

Psychology Research and Behavior Management is an international, peer-reviewed, open access journal focusing on the science of psychology and its application in behavior management to develop improved outcomes in the clinical, educational, sports and business arenas. Specific topics covered in the journal include: Neuroscience, memory and decision making; Behavior modification and management; Clinica applications; Business and sports performance management; Social and developmental studies; Animal studies. The manuscript management system is completely online and includes a very quick and fair peer-review system, which is all easy to use. Visit http://www dovepress.com/testimonials.php to read real quotes from published authors. 